\title{
STABLE MATRICES, THE CAYLEY TRANSFORM, AND CONVERGENT MATRICES
}

\section{TYLER HAYNES}

\author{
Mathematics Department \\ Saginaw Valley State University \\ University Center, Michigan 48710 \\ (Recelved January 17, 1990)
}

\begin{abstract}
The main result is that a square matrix $D$ is convergent $\left(\lim D^{n}=0\right)$ if and $n \rightarrow \infty$

only if it is the Cayley transform $C_{A}=(I-A)^{-1}(I+A)$ of a stable matrix $A$, where a stable matrix is one whose characteristic values all have negative real parts. In passing, the concept of Cayley transform is generalized, and the generalized version is shown closely related to the cquation $A G+G B=D$. This gives rise to a characterization of the non-singularity of the mapping $\mathrm{X} \rightarrow \mathrm{AX}+\mathrm{XB}$. As consequences are derived several characterizations of stability (closely related to Lyapunov's result) which involve Cayley transforms.
\end{abstract}

KEY WORDS AND PHRASES. Stable matrix, Cayley transform, convergent matrix. 1980 AMS SUBJECT CLASSIFICATION CODES. 15A04, 15A24

Both Taussky and Stein [Stein, 1965] have written on the connection between stable matrices and convergent matrices. The link joining the two is the Cayley transform: a matrix is convergent $\Leftrightarrow$ it is the Cayley transform of a stable matrix (theorem 8 ).

Cayley transforms are introduced by considering the matrix equation $\mathrm{AX}+\mathrm{XB}=\mathrm{C}$. But first a lemma:

Lemma 1: Over field $\mathrm{F}$ let matrix $\mathrm{A}$ be $\mathrm{n} \times \mathrm{n}$ and let $\mathrm{x}$ be either indeterminate over $\mathrm{F}$ or in $\mathrm{F}$ but not a characteristic value of $\mathrm{A}$. Then

$$
(x I-A)^{-1}(x I+A)=(x I+A)(x I-A)^{-1} .
$$

If either expression in (1) is denoted by $C_{A, x}$, then $C_{A^{\prime}, x}=C_{A, x}^{\prime}$ If $x \neq 0$, then

$$
A=x\left(C_{A, x}-1\right)\left(C_{A, x}+I\right)^{-1} \text {. }
$$

Proof: Since $x$ is not a characteristic value of $A,(x I-A)^{-1}$ exists. (1) follows from

$$
(x I+A)(x I-A)=(x I-A)(x I+A) \text {. }
$$

Before (2) can be derived, the non-singularity of $\mathrm{C}_{\mathrm{A}, \mathrm{x}}+\mathrm{I}$ must be proven. This equation holds: 


$$
\begin{aligned}
C_{A, x}+I=(x I+A)(x I-A)^{-1}+(x I-A)(x I-A)^{-1} \\
=2 x(x I-A)^{-1} .
\end{aligned}
$$

Therefore, $\left|C_{A, x}+1\right|=2 x|x I-A|^{-1} \neq 0$ since $x \neq 0$ and $|x I-A| \neq 0$ (for $x I-A$ is non-singular); hence, $C_{A, x}+I$ is non-singular. (2) then follows directly.

QED

$C_{A, x}$ of (1) is the generalized Cayley transform of $A$. If $x=1$ is not a characteristic value of $A$, then $C_{A, 1}$ is the Cayley transform of $A$; it will be denoted $C_{A}$. Note that the mapping $A \rightarrow C_{A}$ is bijective from the set of matrices having no characteristic value $=1$ onto those having no characteristic value $=-1$, the inverse transformation being determined by (2).

Theorem 2: Let matrix $A$ be $m \times m, G$ and $D$ be $m \times n$, and $B$ be $n \times n$, all with entries in field $\mathrm{F}$.

$$
A G+G B=D \Leftrightarrow G-C_{A, x} G_{B, x}=-2 x\left(x I_{m}-A\right)^{-1} D\left(x I_{n}-B\right)^{-1},
$$

where $\mathrm{x}$ is either indeterminate over $\mathrm{F}$ or in $\mathrm{F}$ but $\neq 0$ and a characteristic value of ncither $\mathrm{A}$ nor B.

Proof: $x$ satislies the requirements for $\mathrm{C}_{\mathrm{A}, \mathrm{x}}$ and $\mathrm{C}_{\mathrm{B}, \mathrm{x}}$ to exist, according to the lemma, and the dimensions of $\mathrm{C}_{\mathrm{A}, \mathrm{x}}, \mathrm{C}_{\mathrm{B}, \mathrm{x}},\left(\mathrm{xI}_{\mathrm{m}}-\mathrm{A}\right)^{-1}$, and $\left(\mathrm{xI}_{\mathrm{n}}-\mathrm{B}\right)^{-1}$ are such that the expression on the right of (4) is well-defined.

$$
\begin{aligned}
& \mathrm{AG}+\mathrm{GB}=\mathrm{D} \\
& \Leftrightarrow \quad(x G-A G)\left(x_{n}-B\right)-(x G+A G)\left(x_{n}+B\right)=-2 \times D \\
& \Leftrightarrow \quad\left(x_{m}-A\right) G\left(x I_{n}-B\right)-\left(x I_{m}+A\right) G\left(x I_{n}+B\right)=-2 x D \\
& \Leftrightarrow \quad G-\left(x I_{m}-A\right)^{-1}\left(x_{m}+A\right) G\left(x I_{n}+B\right)\left(x I_{n}-B\right)^{-1}=-2 x\left(x I_{m}-A\right)^{-1} D\left(x I_{n}-B\right)^{-1} \\
& \Leftrightarrow \quad G_{-C_{A, x}} G_{B, x}=-2 x\left(x_{m}-A\right)^{-1} D\left(x I_{n}-B\right)^{-1}
\end{aligned}
$$

One consequence of the preceding theorem is the celebrated result that every properly orthogonal“" matrix $\mathrm{P}$ can be expressed as $\mathrm{P}=(\mathrm{I}+\mathrm{K})^{-1}(\mathrm{I}-\mathrm{K})$, where $\mathrm{K}$ is a real skew matrix. To derive it, in the theorem let $F=$ real number field, $G=I, D=O, x=-1$, and $B=A^{\prime}$. Then it follows that $A+A^{\prime}=0 \Leftrightarrow P^{\prime}=I$, where $P=(-I-A)^{-1}(-I+A)=(I+A)^{-1}(I-A)$, the relationship between $\mathrm{P}$ and $\mathrm{A}$ being determined by (1) and (2) of the lemma (cf. the remark on the bijective character of $\left.A \rightarrow C_{A}\right)$. Likewise the Cayley parametrization of unitary matrices follows [Gantmacher, Vol. I; p. 279 (95)].

Over a field $\mathrm{F}$ let $\mathrm{A}$ be an $\mathrm{m} \times \mathrm{m}$ matrix, $\mathrm{X}$ an $\mathrm{m} \times \mathrm{n}$ matrix and $\mathrm{B}$ an $\mathrm{n} \times \mathrm{n}$ matrix. Let $\mathscr{L}_{\mathrm{A}, \mathrm{B}}=\mathrm{AX}+\mathrm{XB}$. Clearly the mapping $\mathcal{L}_{\mathrm{A}, \mathrm{B}}: \quad \mathrm{X} \rightarrow \mathrm{AX}+\mathrm{XB}$ is a linear transformation on the 
lincar space of $m \times n$ matriccs. Denote $\mathcal{L}_{\mathrm{A}, \mathrm{A}^{*}}$ by $\mathcal{L}_{\mathrm{A}}: \mathcal{L}_{\mathrm{A}}(\mathrm{X})=\mathrm{AX}+\mathrm{XA}^{*}$, where all matrices arc of the same dimension.

Corollary 3: Let $\mathrm{A}, \mathrm{B}, \mathrm{G}, \mathrm{x}$, and $\mathrm{F}$ be as in theorem 2. Then the mapping $\mathrm{G} \rightarrow \mathrm{G}-\mathrm{C}_{\mathrm{A}, \mathrm{x}} \mathrm{GC}_{\mathrm{B}, \mathrm{x}}$ is linear from the set of all $\mathrm{m} \times \mathrm{n}$ matrices into itself. This mapping is nonsingular $\Leftrightarrow \mathcal{L}_{\mathrm{A}, \mathrm{B}}$ is non-singular.

Proof: The linearity of the mapping is obvious. $\mathscr{L}_{\mathrm{A}, \mathrm{B}}$ is non-singular $\Leftrightarrow$ for cvery $\mathrm{D}$ there exists a solution of $\mathrm{AX}+\mathrm{XB}=\mathrm{D} \Leftrightarrow$ for every $\mathrm{E}$ there exists a solution of $\mathrm{X}-\mathrm{C}_{\mathrm{A}, \mathrm{X}} \mathrm{XC}_{\mathrm{B}, \mathrm{X}}=$ $E$ (thcorem 2 and the non-singularity of $\mathrm{xI}_{\mathrm{m}}-\mathrm{A}$ and $\mathrm{xI}_{\mathrm{n}}-\mathrm{B}$ ) $\Leftrightarrow$ the mapping $\mathrm{G} \rightarrow \mathrm{G}-\mathrm{C}_{\mathrm{Ax}} \mathrm{GC}_{\mathrm{B}, \mathrm{x}}$ is non-singular.

In the rest of this article, let $\mathrm{F}$ be the field of complex numbers and let all matrices be square.

The inertia of an $n \times n$ matrix $X$ is the ordered triple of integers $(\pi(X), \nu(X) \delta(X))=$ $\operatorname{In}(X)$, where $\pi(X)$ is the number of characteristic values of $X$ whose real parts are positive, $\nu(\mathrm{X})$, the number whose real parts are negative, and $\delta(\mathrm{X})$ the number whose real parts are 0 .

Corollary 4: If $A$ has no characteristic value $=1$, then $\operatorname{In}\left(I-C_{A} C_{A}{ }^{*}\right)=\operatorname{In}\left(-\left(A+A^{*}\right)\right)$.

Proof: $C_{A^{*}}=C_{A}{ }^{*}$ by a slight modification of lemma 1 . In theorem 2 , let $B=A^{*}, G$ $=I$, and $x=1$; then $D=A+A^{*}$. Therefore, $I-C_{A} C_{A}^{*}=I-C_{A} I C_{A^{*}}=-2(I-A)^{-1}\left(A+A^{*}\right)\left(I-A^{*}\right)^{-1}$ $=(I-A)^{-1}\left[-2\left(A+A^{*}\right)\right]\left[(I-A)^{-1}\right]^{*}$. Since the last expression is congruent to $-2\left(A+A^{*}\right)$, their inertias are the same, and $\operatorname{In}\left(-2\left(A+A^{*}\right)\right)=\operatorname{In}\left(-\left(A+A^{*}\right)\right)$.

A square matrix is $\underline{\text { stable }} \Leftrightarrow$ all its characteristic values have negative real parts. $S$ denotes the set of all stable $n \times n$ matrices, II denotes the set of all positive-definite hermitian matrices and $\mathrm{N}$ denotes the set of all negative-definite hermitian matrices.

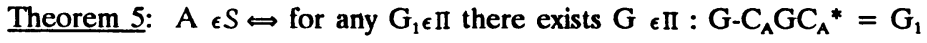
$\Leftrightarrow$ there exists $G_{1} \epsilon \Pi: G_{-} C_{A} G C_{A}{ }^{*}=G_{1}$ for some $G \epsilon \Pi$.

Proof: In theorem 2, let $B=A^{*}, x=1$ (for 1 is not characteristic of a stable matrix and $C_{A}$ presupposes that $\left.x \neq 1\right)$, and $D=-1 / 2(I-A) G_{1}\left(I-A^{*}\right)$. Then the last term of (4) is $G_{1}$, and (4) becomes

$$
A G+G A^{*}=D \Leftrightarrow G \cdot C_{A} G_{A} C^{*}=G_{1} .
$$

$D$ is hermitely congruent to $-1 / 2 G_{1}$, and $\operatorname{so} \operatorname{In}(D)=\operatorname{In}\left(-1 / 2 G_{1}\right)$. Therefore, $G_{1} \in \Pi \Leftrightarrow D \in N$.

First equivalence: Assume $A \in S$. For any $G_{1} \in \Pi, D \in N$. Therefore, $\exists G \in \Pi: A G+G A^{*}$ $=D$ [Taussky], so G- $C_{A} G C_{A}{ }^{*}=G_{1}$. Conversely, if for any $G_{1} \epsilon \Pi$ there exists $G \epsilon \Pi$ : $\mathrm{G}^{-} \mathrm{C}_{A} \mathrm{GC}_{\mathrm{A}}{ }^{*}=\mathrm{G}_{1}$, then $A G+\mathrm{GA}^{*}=\mathrm{D}$; since $\mathrm{G}_{1}$ is arbitrary, so is $\mathrm{D}$, for I-A and I-A* are non- 
singular, otherwise $C_{A}$ and $C_{A}{ }^{*}=C_{A^{*}}$ would not be defined. Since $D \in N, A \in S$ [Taussky].

Second equivalence: Assume $A \in S$. Then $\exists G \in \Pi: A G+G^{*}=D$ for some $D \in N$, and so $G-C_{A} G C_{A}{ }^{*}=G_{1} ; G_{1} \in \Pi$ as above. Conversely, if, for some $G_{1} \in \Pi, G-C_{A} G C_{A}{ }^{*}=G_{1}$ for some $G \in \Pi$, then $A G+G A^{*}=D$ and $D \in N$. Hence, $A \in S$.

QED

Corollary 6: A $\epsilon S \Leftrightarrow \exists G \in \Pi$ : I- $\operatorname{diag}\left(\mathrm{g}_{1}, \ldots, \mathrm{g}_{\mathrm{n}}\right) \in \mathrm{I}$, where $\left\{\mathrm{g}_{\mathrm{1}}\right\}_{1}^{\mathrm{n}}$ are the roots of $\left|\lambda G_{-} C_{A} G C C_{A}{ }^{*}\right|=0$; furthermore, $g_{i}$ is real $(i=1, \ldots, n)$.

Proof: $\Rightarrow$ Assume $A \epsilon S$. By the first equivalence of the preceding theorem $\exists G \in \Pi$ : G- $C_{A} G C_{A}^{*}=I$. Since both $G$ and $C_{A} G C_{A}^{*}$ are hermitian and $G \in \Pi, \exists R: R$ is non-singular and $R^{\prime} G R=I, R^{\prime}\left(C_{A} G C_{A}{ }^{*}\right) R=\operatorname{diag}\left(g_{1}, \ldots, g_{n}\right)$ where $\left\{g_{i}\right\}$ are the roots of $\left|\lambda G-C_{A} G C_{A}{ }^{*}\right|=0$. Then $R^{\prime} R=R^{\prime} I R=R^{\prime}\left(G-C_{A} G C_{A}^{*}\right) R=R^{\prime} G R-R^{\prime}\left(C_{A} G C_{A}^{*}\right) R=I-\operatorname{diag}\left(g_{1}, \ldots, g_{n}\right) . R^{\prime} R \in \Pi$ because $\mathrm{R}^{\prime} \mathrm{GR}=\mathrm{I} \Rightarrow \mathrm{R}^{\cdot-1} \mathbf{R}^{-1}=\mathrm{G} \epsilon \Pi \Rightarrow \mathrm{R}^{\prime} \epsilon \Pi \Rightarrow \mathbf{R}^{\prime} \mathbf{R} \epsilon \Pi$. Therefore, I-diag $\left(\mathrm{g}_{1}, \ldots, \mathrm{g}_{\mathrm{n}}\right) \epsilon \Pi$.

$\Rightarrow$ Since $G$ and $C_{A} G C_{A}{ }^{*}$ are hermitian and $G \epsilon \Pi, \exists R: R$ is non-singular and $R \cdot G R=I$, $R^{\prime}\left(C_{A} G C_{A}^{*}\right) R=\operatorname{diag}\left(g_{1}, \ldots, g_{n}\right)$ where $\left\{g_{i}\right\}$ are the (real) roots of $\left|\lambda G-C_{A} G C_{A}^{*}\right|=0$. Then $R^{\prime-1}\left[I-\operatorname{diag}\left(\mathrm{g}_{1}, \ldots, \mathrm{g}_{\mathrm{n}}\right)\right] \mathrm{R}^{-1}=\mathrm{R}^{\prime-1} \mathrm{R}^{-1}-\mathrm{R}^{\prime-1} \operatorname{diag}\left(\mathrm{g}_{1}, \ldots, \mathrm{g}_{\mathrm{n}}\right) \mathrm{R}^{-1}=\mathrm{G}^{-\mathrm{C}_{\mathrm{A}}} \mathrm{GC}_{\mathrm{A}}{ }^{*} \epsilon \mathrm{I}$. By the second equivalence of the preceding theorem, $A \in S$.

$\mathrm{g}_{\text {, }}$ is real $(\mathrm{i}=1, \ldots, \mathrm{n})$ [Gantmacher, Vol. I; p. 338, thm. 22].

Corollary 7: $A \in S \Leftrightarrow \exists G \in \Pi: g_{i}<1(i=1, \ldots, n)$ where $\left\{g_{i}\right\}_{1}^{n}$ are the characteristic values of $\mathrm{G}^{-1} \mathrm{C}_{\mathrm{A}} \mathrm{GC}_{\mathrm{A}}{ }^{*}$.

Proof: In the preceding corollary, $G$ is non-singular since $G \in \Pi$. Hence, $\left\{g_{1}\right\}_{1}^{n}$, the roots of $\left|\lambda G-C_{A} G C_{A}{ }^{*}\right|=0$, are the characteristic values of $G^{-1} C_{A} G C_{A}^{*}$, for $\left|\lambda G-C_{A} G C_{A}{ }^{*}\right|=0 \Leftrightarrow$ $|G| \cdot\left|\lambda I-G^{-1} C_{A} G C_{A}{ }^{*}\right|=0 . \quad I-\operatorname{diag}\left(g_{1}, \ldots, g_{n}\right) \in \Pi$ is equivalent to $1-g_{1}>0(i-1, \ldots, n)$.

The algebraic properties of the Cayley transform previously developed will be applied to prove theorems about convergent matrices.

The $n \times n$ matrix $A$ is convergent $\Leftrightarrow \lim _{\mathrm{m} \rightarrow \infty} \mathrm{A}^{\mathrm{m}}=0$.

Theorem 8: $D$ is convergent $\Leftrightarrow \exists A \in S: D=C_{A}$.

Proof: $D$ is convergent $\Leftrightarrow D^{*}$ is convergent.

$\Rightarrow$ Assume that $D$ is convergent. Then $D^{*}$ is convergent. By Stein's theorem [Stein, 1952; p. 82, thm. 1] $(\exists G \in \Pi)\left(\exists G_{1} \epsilon \Pi\right): G-D G D^{*}=G_{1}$. Define $A$ by $A=(D-I)(D+I)^{-1}$; then $D=C_{A^{*}}$. By theorem $2, A G+G A^{*}=-1 / 2(I-A) G_{1}\left(I-A^{*}\right)$. Since $-1 / 2(I-A) G_{1}\left(I-A^{*}\right)$ is hermitely congruent to $-G_{1}, A G+G A^{*} \epsilon$ Nand by [Taussky] A $\epsilon S$.

$\in$ Assume that $A \in S$. Then by theorem 5, $(\exists G \in \Pi)\left(\exists G_{1} \in \Pi\right): \quad G-C_{A} G C_{A}{ }^{*}=G_{1}$. By 
Stein's theorem, $C_{A}{ }^{*}$ is convergent, and so $C_{A}$ is convergent.

QED

Corollary 9: $D$ is convergent $\Leftrightarrow\left(\forall G_{1} \epsilon \pi\right)(\exists G \epsilon \Pi):$ G-DGD* $=G_{1}$

$$
\Leftrightarrow\left(\exists G_{1} \epsilon \Pi\right)(\exists G \epsilon \Pi): G-D G D^{*}=G_{1} .
$$

Proof: By the preceding theorem, $D$ is convergent $\Leftrightarrow D=C_{A}$, where $A \epsilon S$. The two equivalences follow from this fact and theorem 5.

QED

The preceding corollary is a theorem of Taussky's [Taussky; p. 7, thm. 5], which is itsclf a strengthening of Stein's theorem.

\section{REFERENCES}

1. Gantmacher, F. R. The Theory of Matrices. 2 vols. Translated by K. A. Hirsch. New York: Chelsea Publishing Company, 1960.

2. Stein, P. "Some General Theorcms on Iterants." Journal of Research of the National Bureau of Standards, Vol. 48, No. 1 (1952 January), 82-83.

3. Stein, P. "On the Ranges of Two Functions of Positive Definite Matrices." Journal of Algebra, Vol. 2, No. 3 (1965 September) 350-353.

4. Taussky, Olga. "Matrices C with $C^{n} \rightarrow 0 . "$ Journal of Algebra, Vol. 1, No. 1 (1964 April), 5-10.

5. Weyl, Hermann. The Classical Groups: Their Invariants and Representations. 2nd ed. Princeton, N.J.: Princeton University Press, 1946. 


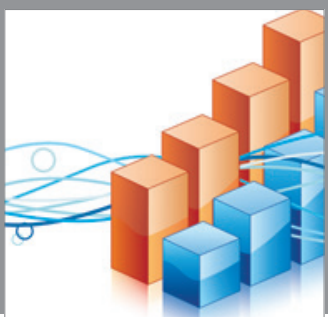

Advances in

Operations Research

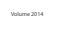

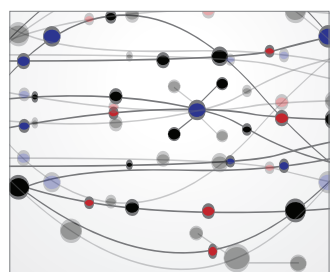

\section{The Scientific} World Journal
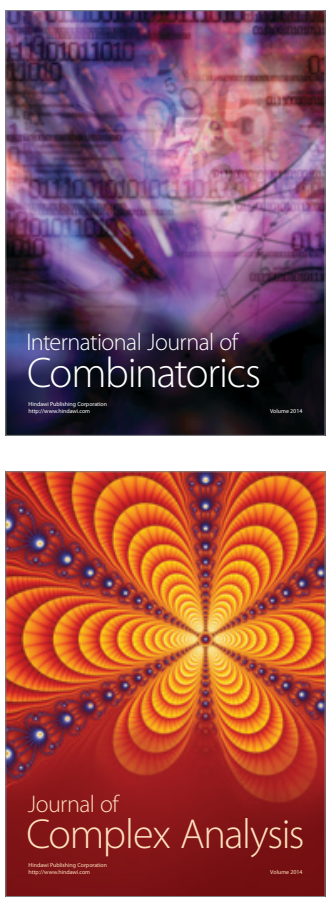

International Journal of

Mathematics and

Mathematical

Sciences
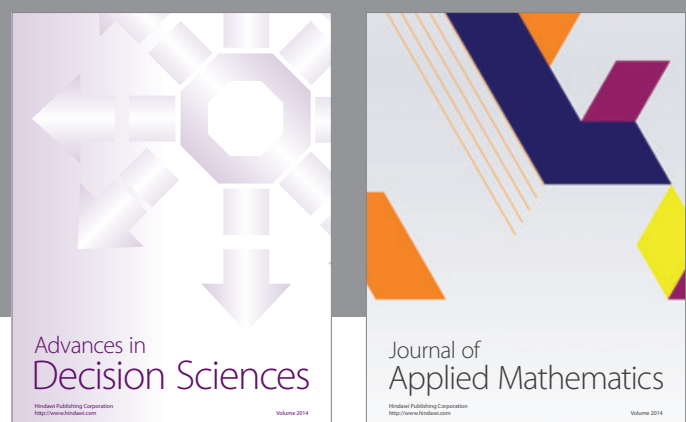

Journal of

Applied Mathematics
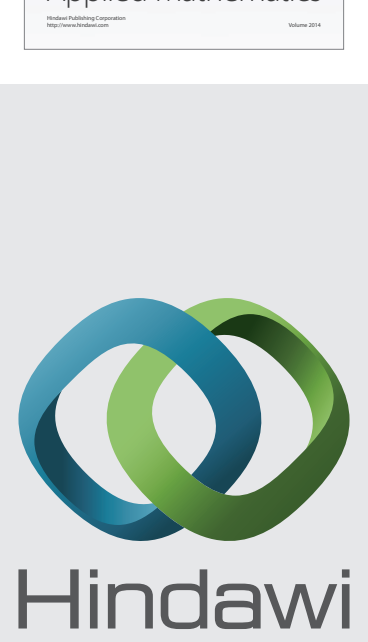

Submit your manuscripts at http://www.hindawi.com
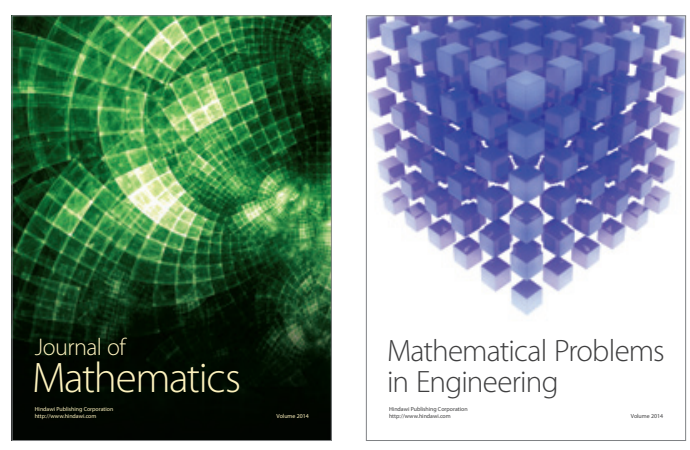

Mathematical Problems in Engineering
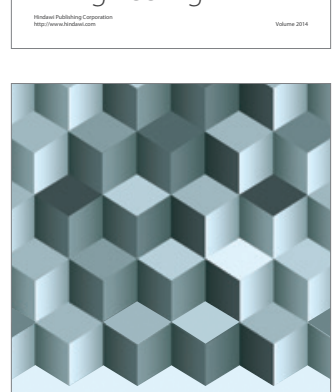

Journal of

Function Spaces
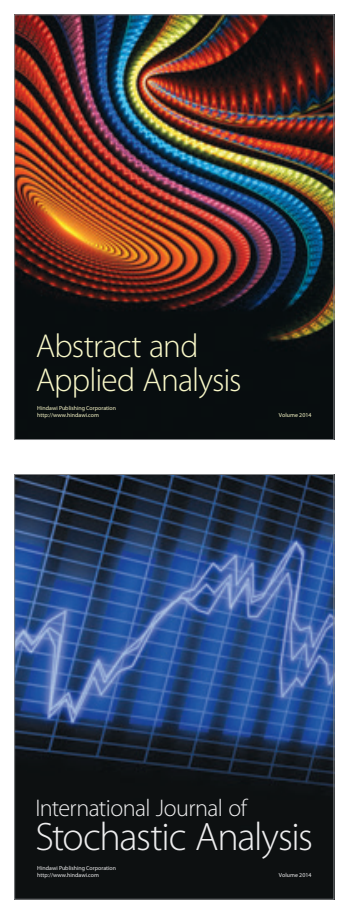

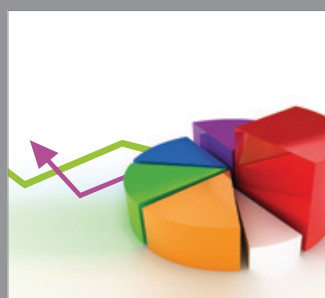

ournal of

Probability and Statistics

Promensencen
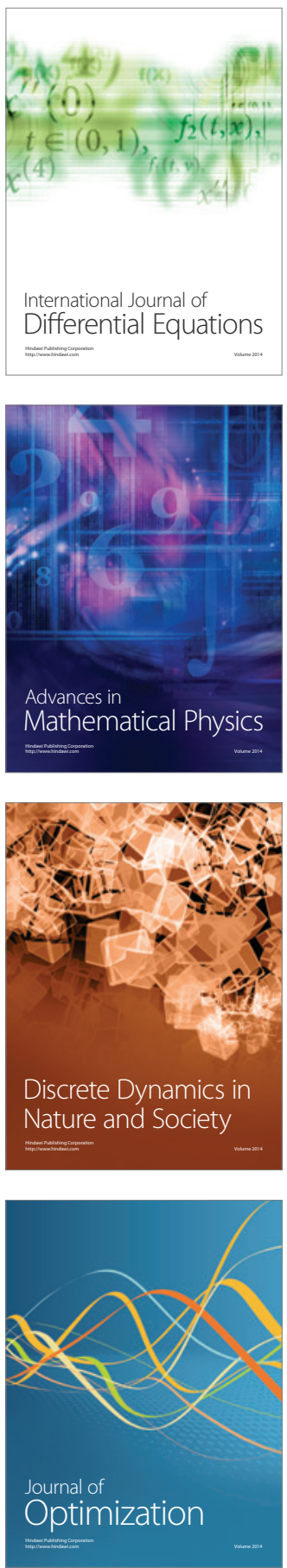